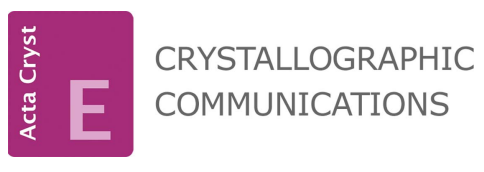

ISSN 2056-9890

Received 5 December 2018

Accepted 22 January 2019

Edited by M. Weil, Vienna University of Technology, Austria

Keywords: crystal structure; ferrocene; planar chirality; boronic acid; ortho-functionalization.

CCDC reference: 1892711

Supporting information: this article has supporting information at journals.iucr.org/e

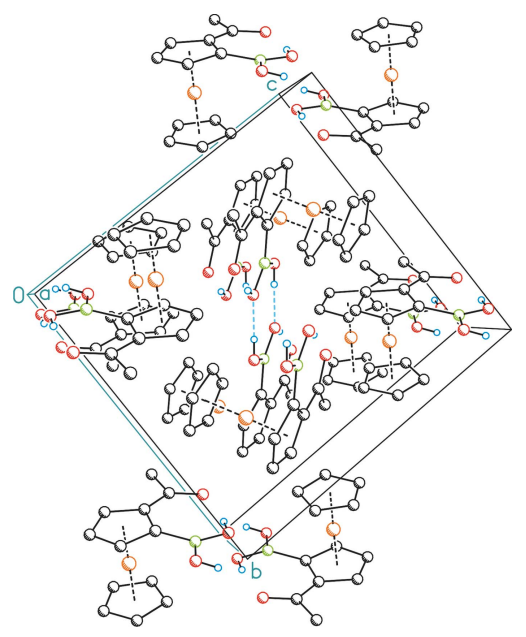

OPEN $\odot$ ACCESS

\section{Crystal structure of (2-acetylferrocen-1-yl)boronic acid}

\author{
Andrea Preuss, Marcus Korb and Heinrich Lang*
}

Technische Universität Chemnitz, Faculty of Natural Sciences, Institute of Chemistry, Inorganic Chemistry, D-09107 Chemnitz, Germany. *Correspondence e-mail: heinrich.lang@chemie.tu-chemnitz.de

(2-Acetylferrocen-1-yl)boronic acid, $\left[\mathrm{Fe}\left(\mathrm{C}_{5} \mathrm{H}_{5}\right)\left(\mathrm{C}_{7} \mathrm{H}_{8} \mathrm{BO}_{3}\right)\right]$ or 2- $\mathrm{C}(\mathrm{O}) \mathrm{CH}_{3}$-1$\mathrm{B}(\mathrm{OH})_{2}-\mathrm{Fc}\left[\mathrm{Fc}=\mathrm{Fe}\left(\eta^{5}-\mathrm{C}_{5} \mathrm{H}_{3}\right)\left(\eta^{5}-\mathrm{C}_{5} \mathrm{H}_{5}\right)\right]$, crystallizes in the centrosymmetric space group $P 2_{1} / n$. The boronic acid functionality interacts via intramolecular hydrogen bonds with the acetyl group and with the $-\mathrm{B}(\mathrm{OH})_{2}$ functionality of an adjacent molecule. The resulting centrosymmetric dimer exhibits an antipositioning of the ferrocenyl moieties towards the central $\mathrm{B}_{2} \mathrm{O}_{4}$ plane. Consequently, an $\left(R_{p}, S_{p}\right)$-, i.e. a meso configuration is present for this dimer. In the crystal, weak $\mathrm{C}-\mathrm{H} \cdots \mathrm{O}$ hydrogen bonds consolidate the molecular packing.

\section{Chemical context}

The synthesis of 1,2-functionalized ferrocenes is a striking topic in ferrocene chemistry (Schaarschmidt \& Lang, 2013; Korb et al., 2014a) and is mostly realized via ortho-directed metalation and subsequent reaction with electrophiles (Schaarschmidt \& Lang, 2013) or intramolecular rearrangement (Werner \& Butenschön, 2013; Korb \& Lang, 2014, 2016; Korb et al., 2017). The resulting ferrocenes are predominantly used as ligands in $C, C$ cross-coupling catalysis (Schaarschmidt et al., 2014; Jensen \& Johannsen, 2003; Vinci et al., 2009; Debono et al., 2010; Karpus et al., 2016), but also the introduction of ferrocenyl substituents by catalytic conversions is of rising interest (Hildebrandt et al., 2011a,b; Speck et al., 2015; Korb et al., 2014b). The introduction of electronically and sterically modified substrates requires the synthesis of the respective ferrocenes that bear groups suitable for oxidative additions or transmetalation reactions (Lehrich et al., 2015; Speck et al., 2014). In case of substrates that are sensitive towards a nucleophilic attack, e.g. acyl groups, the SuzukiMiyaura instead of a Negishi reaction is commonly used, and hence requires the presence of a boronic acid functionality (Speck et al., 2015). However, the acidic protons prevent a straightforward ortho-directed metalation, and additional reaction steps for the introduction and removal of protecting groups are required. Electrophilic aromatic substitution $\left(\mathrm{S}_{\mathrm{E}} \mathrm{Ar}\right)$ reactions are also not suitable, since they usually give 1'- or 3-functionalized products (Rosenblum \& Woodward, 1958).

Within our attempts to synthesize new electronically modified ferrocenes as substrates for Suzuki-Miyaura reactions, we herein present the synthesis and crystal structure of an ortho-functionalized ferrocenylboronic acid, obtained via $\mathrm{S}_{\mathrm{E}} \mathrm{Ar}$ without using a protection group strategy for the acidic protons. 


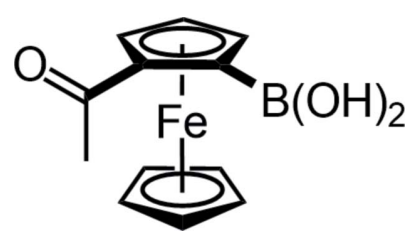

\section{Structural commentary}

The title compound crystallizes in the centrosymmetric space group $P 2_{1} / n$ with one molecule in the asymmetric unit (Fig. 1). An intramolecular hydrogen bond between the oxygen atom of the acetyl group (O1) and the neighbouring hydroxy group (O2) of the boronic acid functionality of 2.650 (2) A (Table 1) is present. Therefore, both substituents are co-planar with each other $\left[\mathrm{BO}_{2} \cdots \mathrm{C}_{2} \mathrm{O}=2.9(4)^{\circ}\right]$. The $\mathrm{C}=\mathrm{O}$ distance of 1.233 (2) $\AA$ is neither affected by the involvement into this hydrogen bond, nor the presence of an ortho substituent and is therefore similar to unsubstituted acetyl ferrocene (Sato et al., 1984).

With regard to the $\mathrm{C}_{5} \mathrm{H}_{3}$ plane of the ferrocenyl backbone, both substituents reveal a slight endo-bending of $7.0(3)^{\circ}$ $\left(\mathrm{C}_{5} \mathrm{H}_{3} \cdots \mathrm{C}_{2} \mathrm{O}\right)$ and $9.5(3)^{\circ}\left(\mathrm{C}_{5} \mathrm{H}_{3} \cdots \mathrm{BO}_{2}\right)$. The ferrocenyl backbone exhibits an eclipsed conformation $(\mathrm{C} 1-\mathrm{Cg}-\mathrm{Cg}-$ $\mathrm{C} 8=8.21(14)^{\circ} ; \mathrm{Cg}$ is the centroid of the respective cyclopentadienyl ring) and a tilt angle of $179.28(2)^{\circ}$. The hydrogen atom at $\mathrm{O} 3$ is directed away from the ferrocenyl backbone and points to an adjacent molecule.

\section{Supramolecular features}

In addition to the aforementioned intramolecular hydrogen bond between $\mathrm{O} 1$ and $\mathrm{O} 2$, the latter atom is also involved as an acceptor of an intermolecular hydrogen bond with the second hydroxy group (O3) of an adjacent boronic acid

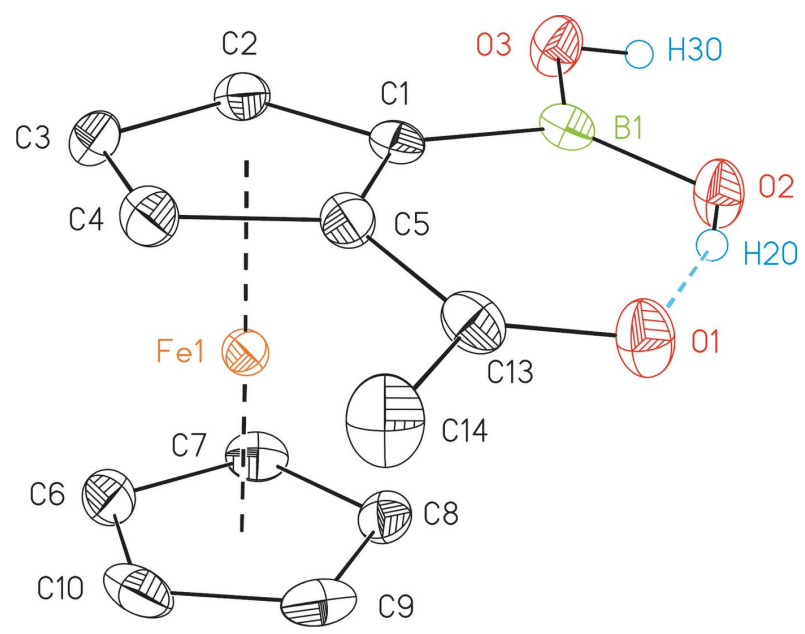

Figure 1

The molecular structure of the title compound showing the intramolecular hydrogen bond between the acetyl and the boronic acid functionalities. Displacement ellipsoids are drawn at the $50 \%$ probability level; C-bonded hydrogen atoms have been omitted for clarity.
Table 1

Hydrogen-bond geometry $\left(\AA,^{\circ}\right)$.

\begin{tabular}{lllll}
\hline$D-\mathrm{H} \cdots A$ & $D-\mathrm{H}$ & $\mathrm{H} \cdots A$ & $D \cdots A$ & $D-\mathrm{H} \cdots A$ \\
\hline $\mathrm{O} 2-\mathrm{H} 2 O \cdots \mathrm{O} 1$ & 0.82 & 1.85 & $2.650(2)$ & 166 \\
$\mathrm{O}^{\mathrm{C}}-\mathrm{H} 3 O \cdots \mathrm{O} 2^{\mathrm{i}}$ & 0.82 & 1.94 & $2.744(2)$ & 168 \\
$\mathrm{C}^{2}-\mathrm{H} 9 \cdots 3^{\mathrm{ii}}$ & 0.93 & 2.45 & $3.308(3)$ & 154 \\
$\mathrm{C} 10-\mathrm{H} 10 \cdots \mathrm{O} 1^{\mathrm{iii}}$ & 0.93 & 2.53 & $3.404(3)$ & 156 \\
\hline
\end{tabular}

Symmetry codes: (i) $-x+1,-y+1,-z+1$; (ii) $x-1, y, z$; (iii) $x-\frac{1}{2},-y+\frac{3}{2}, z-\frac{1}{2}$.

functionality of 2.744 (2) $\AA$ (Fig. 2, Table 1). The resulting dimer is centrosymmetric with the inversion center located at the middle of the eight-membered ring formed by the two boronic acid functionalities. Therefore, both ferrocenyl moieties are positioned anti with regard to the central $\mathrm{B}_{2} \mathrm{O}_{4}$ plane. Hence, a racemic mixture of both enantiomers crystallized, giving the $R_{p} / S_{p}$-configured, i.e. meso diastereomer if the dimer is considered as one supramolecular entity. The respective racem configuration $\left(R_{p} / R_{p}\right.$ or $\left.S_{p} / S_{p}\right)$ is not present within the packing (Fig. 3).

The $\mathrm{B}-\mathrm{O}$ bond lengths involving $\mathrm{O} 3[1.356$ (3) $\mathrm{A}]$ and $\mathrm{O} 2$ [1.362 (3) $\AA]$ are similar, although the latter also acts as a hydrogen-bond acceptor, in contrast to $\mathrm{O} 3$.

A short contact of 4.6807 (14) $\AA$ between a $\mathrm{C}_{5} \mathrm{H}_{3}$ and a $\mathrm{C}_{5} \mathrm{H}_{5}$ ring does not show a perpendicular positioning of the two groups $\left(\beta=25^{\circ}\right)$ and therefore does not fit the criteria for a $T$-shaped $\pi-\pi$ interaction (Sinnokrot et al., 2002). However, weak $\mathrm{C}-\mathrm{H} \cdots \mathrm{O}$ interactions between aromatic $\mathrm{H}$ atoms and the carbonyl $\mathrm{O} 1$ atom and a boronic acid $\mathrm{O}$ atom (O3) consolidate the crystal packing (Table 1).

\section{Database survey}

Besides ferrocenyl boronic acid (Bresner et al., 2004) and acetyl ferrocene (Sato et al., 1984) that are frequently used in

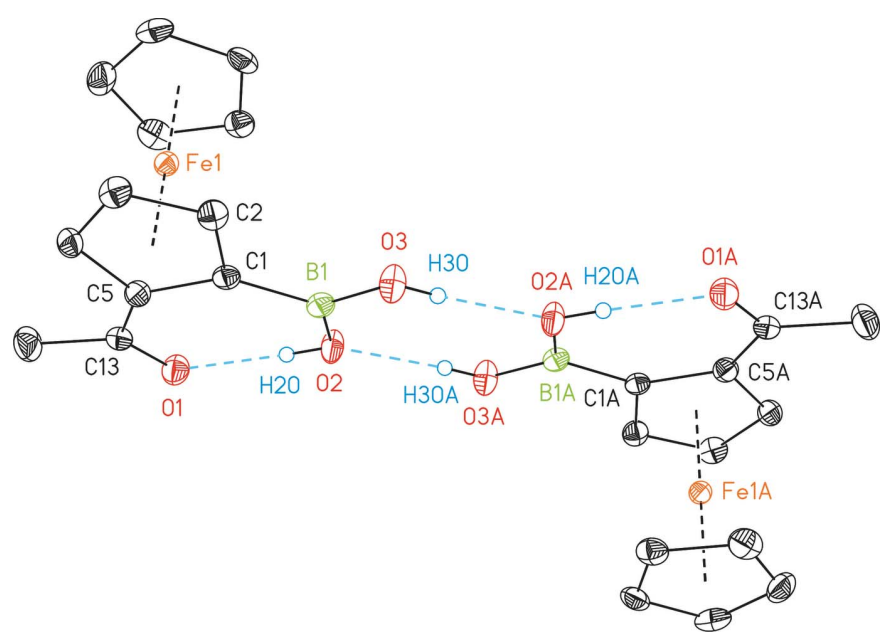

Figure 2

Intra- and intermolecular hydrogen bonds within the dimer, with displacement ellipsoids drawn at the $50 \%$ probability level. C-bonded hydrogen atoms have been omitted for clarity. [Symmetry code: (A) $1-x$, $1-y, 1-z$. 


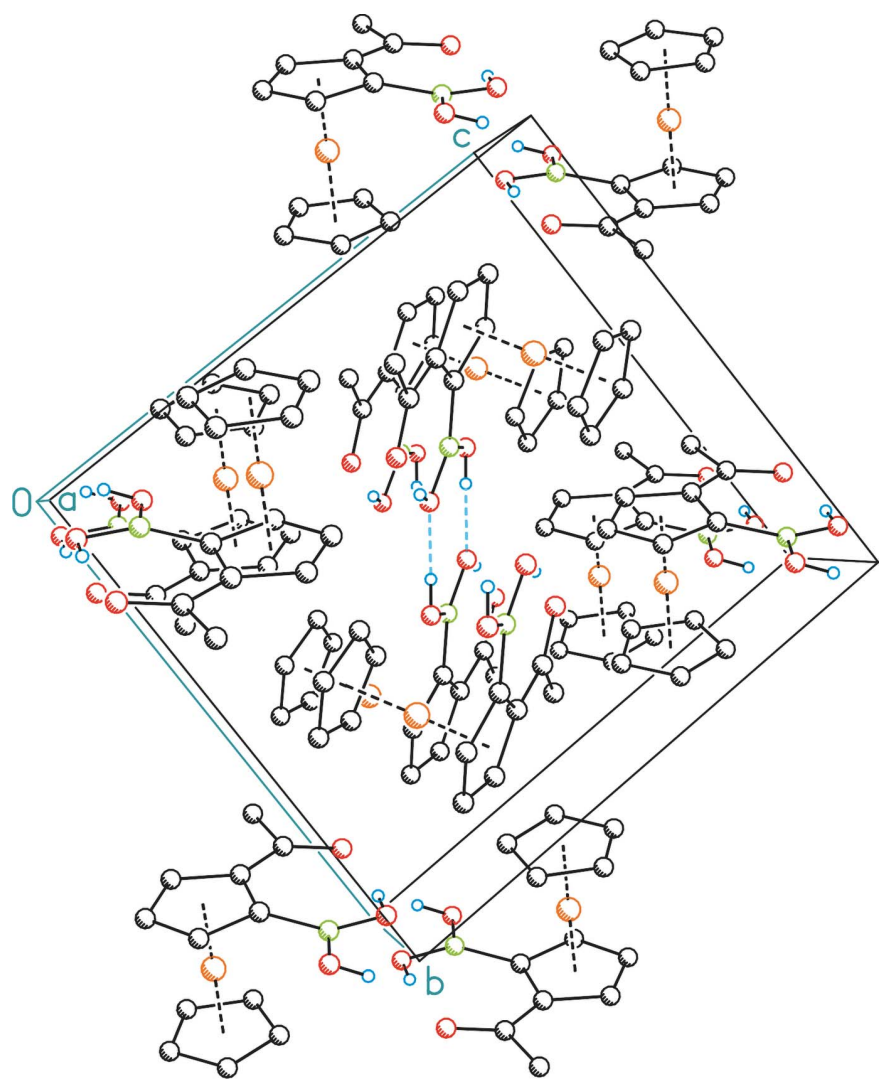

Figure 3

Unit cell of the title compound in a view along [100]. Hydrogen bonds are shown as pale-blue dashed lines; displacement ellipsoids are drawn at the $50 \%$ probability level. C-bonded hydrogen atoms have been omitted for clarity.

general, other ortho-substituted analogues are sparsely described.

Crystal structures of acetylferrocenes bearing additional ortho-substituents are limited to a few examples, e.g. with $\mathrm{PPh}_{2}$ (Torres et al., 2011), iodine (Ferber et al., 2007) and a ferrocenylmethyl group (Xie et al., 2011) as the sole second substituent. In contrast, carbonyl, i.e. formyl or acyl groups, are more common, e.g. in ferrocenoyl methylferrocene (Enders et al., 2003).

Functionalized ferrocenylboronic acids are usually reported together with their protected 1,3,2-dioxaborolane derivatives. As ortho-substituents, diisopropylcarbamoyl (Batsanov et al., 2007) and dimethylcarbamoyl (Norrild \& Søtofte, 2001), together with their respective aminomethyl derivatives (Batsanov et al., 2007; Norrild \& Søtofte, 2001) have structurally been described. Heterocycles, such as imidazolidone (Metallinos et al., 2012) are also known as ortho-substituents for ferrocenyl derivatives.

In case of non-ferrocenyl-based aromatics, the 2- $\mathrm{C}(\mathrm{O}) \mathrm{CH}_{3}-$ $1-\mathrm{B}(\mathrm{OH})_{2}$ substitution pattern is solely reported for the benzene core (Ganguly et al., 2003). In contrast to the title compound, the boronic acid functionality is rotated out of coplanarity with the benzene core and the acetyl group by 78.2 and $77.7^{\circ}$, respectively.
Table 2

Experimental details.

\begin{tabular}{|c|c|}
\hline \multicolumn{2}{|l|}{ Crystal data } \\
\hline Chemical formula & {$\left[\mathrm{Fe}\left(\mathrm{C}_{5} \mathrm{H}_{5}\right)\left(\mathrm{C}_{7} \mathrm{H}_{8} \mathrm{BO}_{3}\right)\right]$} \\
\hline$M_{\mathrm{r}}$ & 271.88 \\
\hline Crystal system, space group & Monoclinic, $P 2_{1} / n$ \\
\hline Temperature $(\mathrm{K})$ & 116 \\
\hline$a, b, c(\AA)$ & $7.7627(3), 11.7335(5), 12.7969(5)$ \\
\hline$\beta\left({ }^{\circ}\right)$ & 98.527 (4) \\
\hline$V\left(\AA^{3}\right)$ & $1152.70(8)$ \\
\hline$Z$ & 4 \\
\hline Radiation type & Мо $K \alpha$ \\
\hline$\mu\left(\mathrm{mm}^{-1}\right)$ & 1.30 \\
\hline Crystal size $(\mathrm{mm})$ & $0.40 \times 0.25 \times 0.20$ \\
\hline \multicolumn{2}{|l|}{ Data collection } \\
\hline Diffractometer & Oxford Gemini S \\
\hline Absorption correction & $\begin{array}{l}\text { Multi-scan (CrysAlis PRO; Rigaku } \\
\text { OD, 2015) }\end{array}$ \\
\hline$T_{\min }, T_{\max }$ & $0.868,1.000$ \\
\hline $\begin{array}{l}\text { No. of measured, independent and } \\
\text { observed }[I>2 \sigma(I)] \text { reflections }\end{array}$ & $4556,2406,2108$ \\
\hline$R_{\text {int }}$ & 0.019 \\
\hline$(\sin \theta / \lambda)_{\max }\left(\AA^{-1}\right)$ & 0.667 \\
\hline \multicolumn{2}{|l|}{ Refinement } \\
\hline$R\left[F^{2}>2 \sigma\left(F^{2}\right)\right], w R\left(F^{2}\right), S$ & $0.030,0.071,1.08$ \\
\hline No. of reflections & 2406 \\
\hline No. of parameters & 157 \\
\hline H-atom treatment & $\mathrm{H}$-atom parameters constrained \\
\hline$\Delta \rho_{\max }, \Delta \rho_{\min }\left(\mathrm{e} \AA^{-3}\right)$ & $0.37,-0.28$ \\
\hline
\end{tabular}

Computer programs: CrysAlis CCD and CrysAlis RED (Oxford Diffraction, 2014), SHELXT2013 (Sheldrick, 2015a), SHELXL2013 (Sheldrick, 2015b), ORTEP-3 for Windows and WinGX (Farrugia, 2012), SHELXTL (Sheldrick, 2008) and publCIF (Westrip, 2010).

For ortho-carbonyl groups in general, the involvement of the boronic acid functionality in inter- and intramolecular hydrogen bonds, similar to the title compound, is a common feature (Yan et al., 2003; Luliński et al., 2007; Durka et al., 2014; Madura et al., 2015).

\section{Synthesis and crystallization}

Ferroceneboronic acid $(0.5 \mathrm{~g}, 2.175 \mathrm{mmol})$ was suspended in acetic anhydride $(10 \mathrm{ml})$. To this suspension $\mathrm{BF}_{3} \cdot \mathrm{OEt}_{2}$ $(0.40 \mathrm{ml}, 3.15 \mathrm{mmol})$ was added in a single portion. The reaction mixture was stirred for $30 \mathrm{~min}$ at ambient temperature. Afterwards, the mixture was poured into ice and was stirred for 10 minutes. A $\mathrm{KOH}$ solution $(9 M, 10 \mathrm{ml})$ was added in a single portion following a neutralization with $\mathrm{K}_{2} \mathrm{CO}_{3}$ until the $\mathrm{CO}_{2}$ evolution stopped. The mixture was extracted with dichloromethane $(3 \times 20 \mathrm{ml})$ and the organic phase was dried over $\mathrm{MgSO}_{4}$. The volatiles were removed in vacuum (1 mbar). The crude material obtained was purified by flash chromatography on silica using a $4 / 1(v / v)$ diethyl ether/ dichloromethane mixture. The title compound was isolated as a brown solid. Yield: $75 \mathrm{mg}(0.28 \mathrm{mmol}, 13 \%$ based on ferroceneboronic acid).

IR data (KBr, v/cm $\left.{ }^{-1}\right): 3357(w), 2925(m), 2855(m), 1685$ $(\mathrm{m}), 1654(\mathrm{~s}), 1647(\mathrm{~m}), 1636(\mathrm{~s}), 1618(\mathrm{~s}), 1578(\mathrm{~m}), 1559(\mathrm{~m})$, $1522(\mathrm{~m}), 1507(\mathrm{~m}), 1457(\mathrm{~s}) ; 1419(\mathrm{~s}), 1411(\mathrm{~s}), 1374(\mathrm{~s}), 1354$ $(s), 1345(s) ; 1318(m), 1247(m), 1207(m), 1134(m), 1106(m)$, $1094(m), 1045(m), 1001(w), 924(w), 873(w), 862(w), 785$ 
$(w), 668(m), 642(w) .{ }^{1} \mathrm{H}$ NMR $\left(\mathrm{CDCl}_{3}, \delta\right): 2.49\left(s, 3 \mathrm{H}, \mathrm{CH}_{3}\right)$, $4.23\left(s, 5 \mathrm{H}, \mathrm{C}_{5} \mathrm{H}_{5}\right), 4.78\left(t, J_{\mathrm{HH}}=2.6 \mathrm{~Hz}, 1 \mathrm{H}, \mathrm{C}_{5} \mathrm{H}_{3}\right), 4.92(d d$, $\left.J_{\mathrm{HH}}=2.6 \mathrm{~Hz}, 1.3 \mathrm{~Hz}, 1 \mathrm{H}, \mathrm{C}_{5} \mathrm{H}_{3}\right), 5.01\left(d d, J_{\mathrm{HH}}=2.6 \mathrm{~Hz}, 1.3 \mathrm{~Hz}\right.$, $\left.\left.1 \mathrm{H}, \mathrm{C}_{5} \mathrm{H}_{3}\right), 7.38\left(b r s, 2 \mathrm{H}, \mathrm{B}(\mathrm{OH})_{2}\right) \cdot{ }^{13} \mathrm{C}_{\{}{ }^{1} \mathrm{H}\right\}$ NMR $\left(\mathrm{CDCl}_{3}, \delta\right)$ : $28.1\left(\mathrm{CH}_{3}\right), 71.1\left(\mathrm{C}_{5} \mathrm{H}_{5}\right), 76.1\left(\mathrm{C}_{5} \mathrm{H}_{3}\right), 77.2\left(\mathrm{C}_{5} \mathrm{H}_{3}\right), 80.1\left(\mathrm{C}_{5} \mathrm{H}_{3}\right)$, $81.1\left(\mathrm{C}_{5} \mathrm{H}_{3}\right), 81.8\left(\mathrm{C}_{5} \mathrm{H}_{3}\right), 208.1(\mathrm{CO})$. HRMS (ESI-TOF, $\left.\mathrm{m} / z\right)$. calculated for $\mathrm{C}_{12} \mathrm{H}_{13} \mathrm{BFeO}_{3} 272.0304$, found $272.0320[\mathrm{M}]^{+}$.

Crystals suitable for X-ray crystallography were obtained from evaporation of a saturated dichloromethane solution at ambient temperature.

\section{Refinement}

Crystal data, data collection and structure refinement detail are summarized in Table 2. $\mathrm{C}$-bound $\mathrm{H}$ atoms were placed in calculated positions and constrained to ride on their parent atoms with $U_{\text {iso }}(\mathrm{H})=1.2 U_{\text {eq }}(\mathrm{C})$ and a $\mathrm{C}-\mathrm{H}$ distance of $0.93 \AA$ for aromatic and $U_{\text {iso }}(\mathrm{H})=1.5 U_{\text {eq }}(\mathrm{C})$ and a $\mathrm{C}-\mathrm{H}$ distance of $0.96 \AA$ for methyl $\mathrm{H}$ atoms, with their torsion angle derived from the residual electron density. The hydroxy hydrogen atoms were located from difference-Fourier maps but were treated with idealized geometry with $U_{\text {iso }}(\mathrm{H})=1.5 U_{\text {eq }}(\mathrm{O})$, an $\mathrm{O}-\mathrm{H}$ distance of $0.82 \AA$ and a torsion angle derived from the residual electron density.

\section{Funding information}

We are grateful to the Federal Cluster of Excellence EXC 1075 "MERGE Technologies for Multifunctional Lightweight Structures". This project has received funding from the European Social Fund (ESF). The publication costs of this article were funded by the German Research Foundation/ DFG-392676956 and the Technische Universität Chemnitz in the funding program Open Access Publishing.

\section{References}

Batsanov, A. S., Hérault, D., Howard, J. A. K., Patrick, L. G. F., Probert, M. R. \& Whiting, A. (2007). Organometallics, 26, 24142419.

Bresner, C., Aldridge, S., Fallis, I. A. \& Ooi, L.-L. (2004). Acta Cryst. E60, m441-m443.

Debono, N., Labande, A., Manoury, E., Daran, J.-C. \& Poli, R. (2010). Organometallics, 29, 1879-1882.

Durka, K., Górska, A., Kliś, T., Serwatowski, J. \& Woźniak, K. (2014). Tetrahedron Lett. 55, 1234-1238.

Enders, D., Klumpen, T. \& Raabe, G. (2003). Synlett, pp. 1198-1200. Farrugia, L. J. (2012). J. Appl. Cryst. 45, 849-854.

Ferber, B., Top, S., Herson, P. \& Jaouen, G. (2007). Organometallics, 26, 1686-1691.

Ganguly, A., Meyers, C. Y. \& Robinson, P. D. (2003). Acta Cryst. E59, o759-0761.
Hildebrandt, A., Schaarschmidt, D., Claus, R. \& Lang, H. (2011a). Inorg. Chem. 50, 10623-10632.

Hildebrandt, A., Schaarschmidt, D. \& Lang, H. (2011b). Organometallics, 30, 556-563.

Jensen, J. F. \& Johannsen, M. (2003). Org. Lett. 5, 3025-3028.

Karpus, A., Yesypenko, O., Boiko, V., Poli, R., Daran, J.-C., Voitenko, Z., Kalchenko, V. \& Manoury, E. (2016). Eur. J. Org. Chem. pp. 3386-3394.

Korb, M. \& Lang, H. (2014). Organometallics, 33, 6643-6659.

Korb, M. \& Lang, H. (2016). Inorg. Chem. Commun. 72, 30-32.

Korb, M., Lehrich, S. W. \& Lang, H. (2017). J. Org. Chem. 82, 31023124.

Korb, M., Pfaff, U., Hildebrandt, A., Rüffer, T. \& Lang, H. (2014b). Eur. J. Inorg. Chem. pp. 1051-1061.

Korb, M., Schaarschmidt, D. \& Lang, H. (2014a). Organometallics, 33, 2099-2108.

Lehrich, S. W., Hildebrandt, A., Korb, M. \& Lang, H. (2015). J. Organomet. Chem. 792, 37-45.

Luliński, S., Madura, I., Serwatowski, J., Szatyłowicz, H. \& Zachara, J. (2007). New J. Chem. 31, 144-154.

Madura, I. D., Adamczyk-Woźniak, A. \& Sporzyński, A. (2015). J. Mol. Struct. 1083, 204-211.

Metallinos, C., John, J., Zaifman, J. \& Emberson, K. (2012). Adv. Synth. Catal. 354, 602-606.

Norrild, J. C. \& Søtofte, I. (2001). J. Chem. Soc. Perkin Trans. 2, pp. 727-732.

Oxford Diffraction (2014). CrysAlis CCD and CrysAlis RED. Oxford Diffraction, Abingdon, England.

Rigaku OD (2015). Crys Alis PRO. Rigaku OD, Abingdon, England.

Rosenblum, M. \& Woodward, R. B. (1958). J. Am. Chem. Soc. 80, 5443-5449.

Sato, K., Katada, M., Sano, H. \& Konno, M. (1984). Bull. Chem. Soc. Jpn, 57, 2361-2365.

Schaarschmidt, D., Grumbt, M., Hildebrandt, A. \& Lang, H. (2014). Eur. J. Org. Chem. 2014, 6676-6685.

Schaarschmidt, D. \& Lang, H. (2013). Organometallics, 32, 56685704.

Sheldrick, G. M. (2008). Acta Cryst. A64, 112-122.

Sheldrick, G. M. (2015a). Acta Cryst. A71, 3-8.

Sheldrick, G. M. (2015b). Acta Cryst. C71, 3-8.

Sinnokrot, M. O., Valeev, E. F. \& Sherrill, C. D. (2002). J. Am. Chem. Soc. 124, 10887-10893.

Speck, J. M., Korb, M., Rüffer, T., Hildebrandt, A. \& Lang, H. (2014). Organometallics, 33, 4813-4823.

Speck, J. M., Korb, M., Schade, A., Spange, S. \& Lang, H. (2015). Organometallics, 34, 3788-3798.

Torres, J., Sepúlveda, F., Carrión, M. C., Jalón, F. A., Manzano, B. R., Rodríguez, A. M., Zirakzadeh, A., Weissensteiner, W., Mucientes, A. E. \& Peña, M. A. (2011). Organometallics, 30, 3490-3503.

Vinci, D., Martins, N., Saidi, O., Bacsa, J., Brigas, A. \& Xiao, J. (2009). Can. J. Chem. 87, 171-175.

Werner, G. \& Butenschön, H. (2013). Organometallics, 32, 5798-5809.

Westrip, S. P. (2010). J. Appl. Cryst. 43, 920-925.

Xie, R.-J., Han, L.-M., Zhu, N., Hong, H.-L. \& Suo, Q.-L. (2011). J. Coord. Chem. 64, 3180-3188.

Yan, H., Beatty, A. M. \& Fehlner, T. P. (2003). J. Am. Chem. Soc. 125, 16367-16382. 


\section{supporting information}

Acta Cryst. (2019). E75, 268-271［https://doi.org/10.1107/S2056989019001178]

\section{Crystal structure of (2-acetylferrocen-1-yl)boronic acid}

\section{Andrea Preuss, Marcus Korb and Heinrich Lang}

\section{Computing details}

Data collection: CrysAlis CCD (Oxford Diffraction, 2014); cell refinement: CrysAlis RED (Oxford Diffraction, 2014); data reduction: CrysAlis RED (Oxford Diffraction, 2014); program(s) used to solve structure: SHELXT2013 (Sheldrick, 2015a); program(s) used to refine structure: SHELXL2013 (Sheldrick, 2015b); molecular graphics: ORTEP-3 for Windows (Farrugia, 2012) and SHELXTL (Sheldrick, 2008); software used to prepare material for publication: WinGX (Farrugia, 2012) and publCIF (Westrip, 2010).

\section{(2-Acetylferrocen-1-yl)boronic acid}

\section{Crystal data}

\section{$\left[\mathrm{Fe}\left(\mathrm{C}_{5} \mathrm{H}_{5}\right)\left(\mathrm{C}_{7} \mathrm{H}_{8} \mathrm{BO}_{3}\right)\right]$}

$M_{r}=271.88$

Monoclinic, $P 2_{1} / n$

$a=7.7627(3) \AA$

$b=11.7335(5) \AA$

$c=12.7969(5) \AA$

$\beta=98.527(4)^{\circ}$

$V=1152.70(8) \AA^{3}$

$Z=4$

\section{Data collection}

Oxford Gemini S diffractometer

Radiation source: fine-focus sealed tube Graphite monochromator $\omega$ scans

Absorption correction: multi-scan (CrysAlis PRO; Rigaku OD, 2015)

$T_{\text {min }}=0.868, T_{\max }=1.000$

4556 measured reflections

Refinement

Refinement on $F^{2}$

Least-squares matrix: full

$R\left[F^{2}>2 \sigma\left(F^{2}\right)\right]=0.030$

$w R\left(F^{2}\right)=0.071$

$S=1.08$

2406 reflections

157 parameters

0 restraints

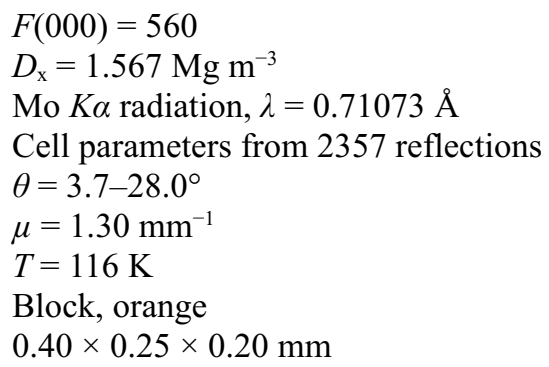

2406 independent reflections

2108 reflections with $I>2 \sigma(I)$

$R_{\text {int }}=0.019$

$\theta_{\max }=28.3^{\circ}, \theta_{\min }=3.2^{\circ}$

$h=-9 \rightarrow 10$

$k=-13 \rightarrow 15$

$l=-16 \rightarrow 16$

2 standard reflections every 50 reflections intensity decay: none

Hydrogen site location: inferred from neighbouring sites

$\mathrm{H}$-atom parameters constrained $w=1 /\left[\sigma^{2}\left(F_{\mathrm{o}}{ }^{2}\right)+(0.028 P)^{2}+0.4245 P\right]$ where $P=\left(F_{\mathrm{o}}{ }^{2}+2 F_{\mathrm{c}}{ }^{2}\right) / 3$

$(\Delta / \sigma)_{\max }=0.001$

$\Delta \rho_{\max }=0.37 \mathrm{e} \AA^{-3}$

$\Delta \rho_{\min }=-0.28$ e $\AA^{-3}$ 


\section{Special details}

Geometry. All esds (except the esd in the dihedral angle between two 1.s. planes) are estimated using the full covariance matrix. The cell esds are taken into account individually in the estimation of esds in distances, angles and torsion angles; correlations between esds in cell parameters are only used when they are defined by crystal symmetry. An approximate (isotropic) treatment of cell esds is used for estimating esds involving l.s. planes.

Refinement. Refinement of $\mathrm{F}^{2}$ against ALL reflections. The weighted $\mathrm{R}$ factor $\mathrm{wR}$ and goodness of fit $\mathrm{S}$ are based on $\mathrm{F}^{2}$, conventional $\mathrm{R}$ factors $\mathrm{R}$ are based on $\mathrm{F}$, with $\mathrm{F}$ set to zero for negative $\mathrm{F}^{2}$. The threshold expression of $\mathrm{F}^{2}>2 \sigma\left(\mathrm{F}^{2}\right)$ is used only for calculating $\mathrm{R}$ factors (gt) etc. and is not relevant to the choice of reflections for refinement. $\mathrm{R}$ factors based on $\mathrm{F}^{2}$ are statistically about twice as large as those based on F, and R factors based on ALL data will be even larger.

Fractional atomic coordinates and isotropic or equivalent isotropic displacement parameters $\left(\AA^{2}\right)$

\begin{tabular}{lllll}
\hline & $x$ & $y$ & $z$ & $U_{\text {iso }} / U_{\text {eq }}$ \\
\hline C1 & $0.2362(2)$ & $0.71679(17)$ & $0.35395(14)$ & $0.0159(4)$ \\
C2 & $0.2734(3)$ & $0.75344(17)$ & $0.25306(15)$ & $0.0189(4)$ \\
$\mathrm{H} 2$ & 0.3701 & 0.7314 & 0.2229 & $0.023^{*}$ \\
$\mathrm{C} 3$ & $0.1414(3)$ & $0.82799(17)$ & $0.20593(16)$ & $0.0202(4)$ \\
$\mathrm{H} 3$ & 0.1361 & 0.8621 & 0.1399 & $0.024^{*}$ \\
$\mathrm{C} 4$ & $0.0188(3)$ & $0.84184(17)$ & $0.27622(15)$ & $0.0193(4)$ \\
$\mathrm{H} 4$ & -0.0804 & 0.8872 & 0.2649 & $0.023^{*}$ \\
$\mathrm{C} 5$ & $0.0743(3)$ & $0.77358(17)$ & $0.36840(14)$ & $0.0174(4)$ \\
$\mathrm{C} 6$ & $-0.0701(3)$ & $0.60243(19)$ & $0.09838(15)$ & $0.0243(5)$ \\
$\mathrm{H} 6$ & -0.0624 & 0.6319 & 0.0318 & $0.029^{*}$ \\
$\mathrm{C} 7$ & $0.0489(3)$ & $0.52589(18)$ & $0.15604(15)$ & $0.0211(4)$ \\
$\mathrm{H} 7$ & 0.1485 & 0.4961 & 0.1339 & $0.025^{*}$ \\
$\mathrm{C} 8$ & $-0.0103(3)$ & $0.50261(18)$ & $0.25363(15)$ & $0.0208(4)$ \\
$\mathrm{H} 8$ & 0.0434 & 0.4547 & 0.3066 & $0.025^{*}$ \\
$\mathrm{C} 9$ & $-0.1650(3)$ & $0.56502(19)$ & $0.25598(17)$ & $0.0249(5)$ \\
$\mathrm{H} 9$ & -0.2307 & 0.5658 & 0.3111 & $0.030^{*}$ \\
$\mathrm{C} 10$ & $-0.2028(3)$ & $0.6261(2)$ & $0.16011(17)$ & $0.0271(5)$ \\
$\mathrm{H} 10$ & -0.2982 & 0.6736 & 0.1410 & $0.033^{*}$ \\
$\mathrm{C} 13$ & $-0.0314(3)$ & $0.75364(18)$ & $0.45290(15)$ & $0.0191(4)$ \\
$\mathrm{C} 14$ & $-0.1979(3)$ & $0.8197(2)$ & $0.44975(17)$ & $0.0286(5)$ \\
$\mathrm{H} 14 \mathrm{~A}$ & -0.2495 & 0.8027 & 0.5117 & $0.043^{*}$ \\
$\mathrm{H} 14 \mathrm{~B}$ & -0.2771 & 0.7988 & 0.3878 & $0.043^{*}$ \\
$\mathrm{H} 14 \mathrm{C}$ & -0.1735 & 0.8998 & 0.4478 & $0.043^{*}$ \\
$\mathrm{~B} 1$ & $0.3493(3)$ & $0.6251(2)$ & $0.42048(17)$ & $0.0180(5)$ \\
$\mathrm{O} 1$ & $0.01228(19)$ & $0.68590(13)$ & $0.52571(11)$ & $0.0242(3)$ \\
$\mathrm{O} 2$ & $0.30364(18)$ & $0.57462(13)$ & $0.50825(10)$ & $0.0224(3)$ \\
$\mathrm{H} 2 \mathrm{O}$ & 0.2091 & 0.5995 & 0.5190 & $0.034^{*}$ \\
$\mathrm{O} 3$ & $0.50175(17)$ & $0.59706(13)$ & $0.38717(11)$ & $0.0237(3)$ \\
$\mathrm{H} 3 \mathrm{O}$ & 0.5472 & 0.5439 & 0.4227 & $0.036^{*}$ \\
$\mathrm{~F} 1$ & $0.03493(3)$ & $0.67352(2)$ & $0.23909(2)$ & $0.01438(10)$ \\
& & & &
\end{tabular}

Atomic displacement parameters $\left(\AA^{2}\right)$

\begin{tabular}{lllllll}
\hline & $U^{11}$ & $U^{22}$ & $U^{33}$ & $U^{12}$ & $U^{13}$ & $U^{23}$ \\
\hline $\mathrm{C} 1$ & $0.0146(9)$ & $0.0161(10)$ & $0.0164(9)$ & $-0.0020(8)$ & $0.0000(7)$ & $-0.0028(8)$ \\
$\mathrm{C} 2$ & $0.0174(10)$ & $0.0184(10)$ & $0.0211(10)$ & $-0.0030(9)$ & $0.0032(8)$ & $0.0000(9)$
\end{tabular}


supporting information

\begin{tabular}{lllllll} 
C3 & $0.0238(11)$ & $0.0167(10)$ & $0.0198(10)$ & $-0.0035(9)$ & $0.0022(8)$ & $0.0045(9)$ \\
C4 & $0.0216(10)$ & $0.0140(10)$ & $0.0218(10)$ & $0.0025(8)$ & $0.0010(8)$ & $-0.0010(9)$ \\
C5 & $0.0191(10)$ & $0.0151(10)$ & $0.0173(9)$ & $0.0011(8)$ & $0.0006(8)$ & $-0.0004(8)$ \\
C6 & $0.0308(12)$ & $0.0261(12)$ & $0.0140(9)$ & $-0.0074(10)$ & $-0.0029(8)$ & $-0.0015(9)$ \\
C7 & $0.0218(10)$ & $0.0192(11)$ & $0.0228(10)$ & $-0.0017(9)$ & $0.0053(8)$ & $-0.0094(9)$ \\
C8 & $0.0256(11)$ & $0.0151(10)$ & $0.0214(10)$ & $-0.0030(9)$ & $0.0020(9)$ & $0.0002(9)$ \\
C9 & $0.0201(11)$ & $0.0265(12)$ & $0.0297(11)$ & $-0.0095(9)$ & $0.0096(9)$ & $-0.0062(10)$ \\
C10 & $0.0176(10)$ & $0.0245(12)$ & $0.0358(12)$ & $-0.0013(10)$ & $-0.0074(9)$ & $-0.0046(10)$ \\
C13 & $0.0192(10)$ & $0.0200(11)$ & $0.0174(9)$ & $0.0015(9)$ & $0.0006(8)$ & $-0.0052(9)$ \\
C14 & $0.0276(12)$ & $0.0328(13)$ & $0.0270(11)$ & $0.0116(10)$ & $0.0096(10)$ & $0.0015(10)$ \\
B1 & $0.0160(11)$ & $0.0185(11)$ & $0.0186(10)$ & $-0.0014(10)$ & $-0.0001(9)$ & $-0.0027(10)$ \\
O1 & $0.0239(8)$ & $0.0309(9)$ & $0.0184(7)$ & $0.0073(7)$ & $0.0048(6)$ & $0.0034(7)$ \\
O2 & $0.0183(7)$ & $0.0286(9)$ & $0.0208(7)$ & $0.0084(7)$ & $0.0048(6)$ & $0.0071(7)$ \\
O3 & $0.0190(7)$ & $0.0253(9)$ & $0.0276(8)$ & $0.0054(7)$ & $0.0059(6)$ & $0.0084(7)$ \\
Fe1 & $0.01391(16)$ & $0.01475(16)$ & $0.01429(15)$ & $0.00022(11)$ & $0.00151(11)$ & $0.00008(11)$ \\
& & & & & & \\
\hline
\end{tabular}

Geometric parameters $\left(A,{ }^{o}\right)$

\begin{tabular}{|c|c|c|c|}
\hline $\mathrm{C} 1-\mathrm{C} 2$ & $1.430(3)$ & $\mathrm{C} 7-\mathrm{Fe} 1$ & $2.043(2)$ \\
\hline $\mathrm{C} 1-\mathrm{C} 5$ & $1.459(3)$ & $\mathrm{C} 7-\mathrm{H} 7$ & 0.9300 \\
\hline $\mathrm{C} 1-\mathrm{B} 1$ & $1.560(3)$ & $\mathrm{C} 8-\mathrm{C} 9$ & $1.411(3)$ \\
\hline $\mathrm{C} 1-\mathrm{Fe} 1$ & $2.0443(18)$ & $\mathrm{C} 8-\mathrm{Fe} 1$ & $2.049(2)$ \\
\hline $\mathrm{C} 2-\mathrm{C} 3$ & $1.413(3)$ & $\mathrm{C} 8-\mathrm{H} 8$ & 0.9300 \\
\hline $\mathrm{C} 2-\mathrm{Fe} 1$ & $2.059(2)$ & $\mathrm{C} 9-\mathrm{C} 10$ & $1.414(3)$ \\
\hline $\mathrm{C} 2-\mathrm{H} 2$ & 0.9300 & $\mathrm{C} 9-\mathrm{Fe} 1$ & $2.043(2)$ \\
\hline $\mathrm{C} 3-\mathrm{C} 4$ & $1.412(3)$ & C9-H9 & 0.9300 \\
\hline $\mathrm{C} 3-\mathrm{Fe} 1$ & $2.062(2)$ & $\mathrm{C} 10-\mathrm{Fe} 1$ & $2.045(2)$ \\
\hline $\mathrm{C} 3-\mathrm{H} 3$ & 0.9300 & $\mathrm{C} 10-\mathrm{H} 10$ & 0.9300 \\
\hline $\mathrm{C} 4-\mathrm{C} 5$ & $1.438(3)$ & $\mathrm{C} 13-\mathrm{O} 1$ & $1.233(2)$ \\
\hline $\mathrm{C} 4-\mathrm{Fe} 1$ & $2.040(2)$ & $\mathrm{C} 13-\mathrm{C} 14$ & $1.503(3)$ \\
\hline $\mathrm{C} 4-\mathrm{H} 4$ & 0.9300 & $\mathrm{C} 14-\mathrm{H} 14 \mathrm{~A}$ & 0.9600 \\
\hline $\mathrm{C} 5-\mathrm{C} 13$ & $1.470(3)$ & $\mathrm{C} 14-\mathrm{H} 14 \mathrm{~B}$ & 0.9600 \\
\hline $\mathrm{C} 5-\mathrm{Fe} 1$ & $2.0150(19)$ & $\mathrm{C} 14-\mathrm{H} 14 \mathrm{C}$ & 0.9600 \\
\hline $\mathrm{C} 6-\mathrm{C} 7$ & $1.415(3)$ & $\mathrm{B} 1-\mathrm{O} 3$ & $1.356(3)$ \\
\hline $\mathrm{C} 6-\mathrm{C} 10$ & $1.416(3)$ & $\mathrm{B} 1-\mathrm{O} 2$ & $1.362(3)$ \\
\hline $\mathrm{C} 6-\mathrm{Fe} 1$ & $2.0414(19)$ & $\mathrm{O} 2-\mathrm{H} 2 \mathrm{O}$ & 0.8200 \\
\hline $\mathrm{C} 6-\mathrm{H} 6$ & 0.9300 & $\mathrm{O} 3-\mathrm{H} 3 \mathrm{O}$ & 0.8200 \\
\hline $\mathrm{C} 7-\mathrm{C} 8$ & $1.420(3)$ & & \\
\hline $\mathrm{C} 2-\mathrm{C} 1-\mathrm{C} 5$ & $105.66(17)$ & $\mathrm{O} 1-\mathrm{C} 13-\mathrm{C} 5$ & $122.50(18)$ \\
\hline $\mathrm{C} 2-\mathrm{C} 1-\mathrm{B} 1$ & $121.86(17)$ & $\mathrm{O} 1-\mathrm{C} 13-\mathrm{C} 14$ & $119.45(18)$ \\
\hline $\mathrm{C} 5-\mathrm{C} 1-\mathrm{B} 1$ & $132.22(17)$ & $\mathrm{C} 5-\mathrm{C} 13-\mathrm{C} 14$ & $118.04(17)$ \\
\hline $\mathrm{C} 2-\mathrm{C} 1-\mathrm{Fe} 1$ & $70.16(11)$ & $\mathrm{C} 13-\mathrm{C} 14-\mathrm{H} 14 \mathrm{~A}$ & 109.5 \\
\hline $\mathrm{C} 5-\mathrm{C} 1-\mathrm{Fe} 1$ & $67.87(10)$ & $\mathrm{C} 13-\mathrm{C} 14-\mathrm{H} 14 \mathrm{~B}$ & 109.5 \\
\hline $\mathrm{B} 1-\mathrm{C} 1-\mathrm{Fe} 1$ & $121.98(14)$ & $\mathrm{H} 14 \mathrm{~A}-\mathrm{C} 14-\mathrm{H} 14 \mathrm{~B}$ & 109.5 \\
\hline $\mathrm{C} 3-\mathrm{C} 2-\mathrm{C} 1$ & $109.94(18)$ & $\mathrm{C} 13-\mathrm{C} 14-\mathrm{H} 14 \mathrm{C}$ & 109.5 \\
\hline $\mathrm{C} 3-\mathrm{C} 2-\mathrm{Fe} 1$ & $70.07(11)$ & $\mathrm{H} 14 \mathrm{~A}-\mathrm{C} 14-\mathrm{H} 14 \mathrm{C}$ & 109.5 \\
\hline $\mathrm{C} 1-\mathrm{C} 2-\mathrm{Fe} 1$ & $69.05(11)$ & $\mathrm{H} 14 \mathrm{~B}-\mathrm{C} 14-\mathrm{H} 14 \mathrm{C}$ & 109.5 \\
\hline
\end{tabular}




\begin{tabular}{|c|c|c|c|}
\hline $\mathrm{C} 3-\mathrm{C} 2-\mathrm{H} 2$ & 125.0 & $\mathrm{O} 3-\mathrm{B} 1-\mathrm{O} 2$ & 119.99 (19) \\
\hline $\mathrm{C} 1-\mathrm{C} 2-\mathrm{H} 2$ & 125.0 & $\mathrm{O} 3-\mathrm{B} 1-\mathrm{C} 1$ & $116.25(18)$ \\
\hline $\mathrm{Fe} 1-\mathrm{C} 2-\mathrm{H} 2$ & 127.5 & $\mathrm{O} 2-\mathrm{B} 1-\mathrm{C} 1$ & $123.75(18)$ \\
\hline $\mathrm{C} 4-\mathrm{C} 3-\mathrm{C} 2$ & $108.30(18)$ & $\mathrm{B} 1-\mathrm{O} 2-\mathrm{H} 2 \mathrm{O}$ & 109.5 \\
\hline $\mathrm{C} 4-\mathrm{C} 3-\mathrm{Fe} 1$ & $69.01(12)$ & $\mathrm{B} 1-\mathrm{O} 3-\mathrm{H} 3 \mathrm{O}$ & 109.5 \\
\hline $\mathrm{C} 2-\mathrm{C} 3-\mathrm{Fe} 1$ & $69.84(11)$ & $\mathrm{C} 5-\mathrm{Fe} 1-\mathrm{C} 4$ & $41.54(8)$ \\
\hline $\mathrm{C} 4-\mathrm{C} 3-\mathrm{H} 3$ & 125.9 & $\mathrm{C} 5-\mathrm{Fe} 1-\mathrm{C} 6$ & $162.67(9)$ \\
\hline $\mathrm{C} 2-\mathrm{C} 3-\mathrm{H} 3$ & 125.9 & $\mathrm{C} 4-\mathrm{Fe} 1-\mathrm{C} 6$ & $124.81(9)$ \\
\hline $\mathrm{Fe} 1-\mathrm{C} 3-\mathrm{H} 3$ & 126.9 & $\mathrm{C} 5-\mathrm{Fe} 1-\mathrm{C} 7$ & $155.19(8)$ \\
\hline $\mathrm{C} 3-\mathrm{C} 4-\mathrm{C} 5$ & $108.07(18)$ & $\mathrm{C} 4-\mathrm{Fe} 1-\mathrm{C} 7$ & $162.14(8)$ \\
\hline $\mathrm{C} 3-\mathrm{C} 4-\mathrm{Fe} 1$ & $70.72(12)$ & $\mathrm{C} 6-\mathrm{Fe} 1-\mathrm{C} 7$ & $40.53(8)$ \\
\hline $\mathrm{C} 5-\mathrm{C} 4-\mathrm{Fe} 1$ & $68.32(11)$ & $\mathrm{C} 5-\mathrm{Fe} 1-\mathrm{C} 9$ & $107.54(8)$ \\
\hline $\mathrm{C} 3-\mathrm{C} 4-\mathrm{H} 4$ & 126.0 & $\mathrm{C} 4-\mathrm{Fe} 1-\mathrm{C} 9$ & $120.33(9)$ \\
\hline $\mathrm{C} 5-\mathrm{C} 4-\mathrm{H} 4$ & 126.0 & $\mathrm{C} 6-\mathrm{Fe} 1-\mathrm{C} 9$ & $68.20(9)$ \\
\hline $\mathrm{Fe} 1-\mathrm{C} 4-\mathrm{H} 4$ & 126.6 & $\mathrm{C} 7-\mathrm{Fe} 1-\mathrm{C} 9$ & $68.08(8)$ \\
\hline $\mathrm{C} 4-\mathrm{C} 5-\mathrm{C} 1$ & $108.02(17)$ & $\mathrm{C} 5-\mathrm{Fe} 1-\mathrm{C} 1$ & $42.11(8)$ \\
\hline $\mathrm{C} 4-\mathrm{C} 5-\mathrm{C} 13$ & $124.06(18)$ & $\mathrm{C} 4-\mathrm{Fe} 1-\mathrm{C} 1$ & $70.05(8)$ \\
\hline $\mathrm{C} 1-\mathrm{C} 5-\mathrm{C} 13$ & $127.32(17)$ & $\mathrm{C} 6-\mathrm{Fe} 1-\mathrm{C} 1$ & $153.94(9)$ \\
\hline $\mathrm{C} 4-\mathrm{C} 5-\mathrm{Fe} 1$ & $70.14(11)$ & $\mathrm{C} 7-\mathrm{Fe} 1-\mathrm{C} 1$ & $119.38(8)$ \\
\hline $\mathrm{C} 1-\mathrm{C} 5-\mathrm{Fe} 1$ & $70.02(11)$ & $\mathrm{C} 9-\mathrm{Fe} 1-\mathrm{C} 1$ & $126.18(8)$ \\
\hline $\mathrm{C} 13-\mathrm{C} 5-\mathrm{Fe} 1$ & $118.56(14)$ & $\mathrm{C} 5-\mathrm{Fe} 1-\mathrm{C} 10$ & $125.46(9)$ \\
\hline $\mathrm{C} 7-\mathrm{C} 6-\mathrm{C} 10$ & $107.85(18)$ & $\mathrm{C} 4-\mathrm{Fe} 1-\mathrm{C} 10$ & $107.24(9)$ \\
\hline $\mathrm{C} 7-\mathrm{C} 6-\mathrm{Fe} 1$ & $69.81(11)$ & $\mathrm{C} 6-\mathrm{Fe} 1-\mathrm{C} 10$ & $40.54(9)$ \\
\hline $\mathrm{C} 10-\mathrm{C} 6-\mathrm{Fe} 1$ & $69.88(11)$ & $\mathrm{C} 7-\mathrm{Fe} 1-\mathrm{C} 10$ & $68.05(9)$ \\
\hline $\mathrm{C} 7-\mathrm{C} 6-\mathrm{H} 6$ & 126.1 & $\mathrm{C} 9-\mathrm{Fe} 1-\mathrm{C} 10$ & $40.46(9)$ \\
\hline $\mathrm{C} 10-\mathrm{C} 6-\mathrm{H} 6$ & 126.1 & $\mathrm{C} 1-\mathrm{Fe} 1-\mathrm{C} 10$ & $163.81(9)$ \\
\hline $\mathrm{Fe} 1-\mathrm{C} 6-\mathrm{H} 6$ & 125.8 & $\mathrm{C} 5-\mathrm{Fe} 1-\mathrm{C} 8$ & $120.19(8)$ \\
\hline $\mathrm{C} 6-\mathrm{C} 7-\mathrm{C} 8$ & $108.03(18)$ & $\mathrm{C} 4-\mathrm{Fe} 1-\mathrm{C} 8$ & $155.41(8)$ \\
\hline $\mathrm{C} 6-\mathrm{C} 7-\mathrm{Fe} 1$ & $69.65(12)$ & $\mathrm{C} 6-\mathrm{Fe} 1-\mathrm{C} 8$ & $68.21(8)$ \\
\hline $\mathrm{C} 8-\mathrm{C} 7-\mathrm{Fe} 1$ & $69.91(11)$ & $\mathrm{C} 7-\mathrm{Fe} 1-\mathrm{C} 8$ & $40.60(8)$ \\
\hline $\mathrm{C} 6-\mathrm{C} 7-\mathrm{H} 7$ & 126.0 & $\mathrm{C} 9-\mathrm{Fe} 1-\mathrm{C} 8$ & $40.32(8)$ \\
\hline $\mathrm{C} 8-\mathrm{C} 7-\mathrm{H} 7$ & 126.0 & $\mathrm{C} 1-\mathrm{Fe} 1-\mathrm{C} 8$ & $107.50(8)$ \\
\hline $\mathrm{Fe} 1-\mathrm{C} 7-\mathrm{H} 7$ & 126.0 & $\mathrm{C} 10-\mathrm{Fe} 1-\mathrm{C} 8$ & $67.96(9)$ \\
\hline $\mathrm{C} 9-\mathrm{C} 8-\mathrm{C} 7$ & $107.85(18)$ & $\mathrm{C} 5-\mathrm{Fe} 1-\mathrm{C} 2$ & $68.80(8)$ \\
\hline $\mathrm{C} 9-\mathrm{C} 8-\mathrm{Fe} 1$ & $69.61(12)$ & $\mathrm{C} 4-\mathrm{Fe} 1-\mathrm{C} 2$ & $67.92(8)$ \\
\hline $\mathrm{C} 7-\mathrm{C} 8-\mathrm{Fe} 1$ & $69.49(12)$ & $\mathrm{C} 6-\mathrm{Fe} 1-\mathrm{C} 2$ & $119.92(8)$ \\
\hline $\mathrm{C} 9-\mathrm{C} 8-\mathrm{H} 8$ & 126.1 & $\mathrm{C} 7-\mathrm{Fe} 1-\mathrm{C} 2$ & $108.35(8)$ \\
\hline $\mathrm{C} 7-\mathrm{C} 8-\mathrm{H} 8$ & 126.1 & $\mathrm{C} 9-\mathrm{Fe} 1-\mathrm{C} 2$ & $164.08(8)$ \\
\hline $\mathrm{Fe} 1-\mathrm{C} 8-\mathrm{H} 8$ & 126.4 & $\mathrm{C} 1-\mathrm{Fe} 1-\mathrm{C} 2$ & $40.79(7)$ \\
\hline $\mathrm{C} 8-\mathrm{C} 9-\mathrm{C} 10$ & $108.23(19)$ & $\mathrm{C} 10-\mathrm{Fe} 1-\mathrm{C} 2$ & $154.21(9)$ \\
\hline $\mathrm{C} 8-\mathrm{C} 9-\mathrm{Fe} 1$ & $70.06(12)$ & $\mathrm{C} 8-\mathrm{Fe} 1-\mathrm{C} 2$ & $126.99(8)$ \\
\hline $\mathrm{C} 10-\mathrm{C} 9-\mathrm{Fe} 1$ & $69.85(12)$ & $\mathrm{C} 5-\mathrm{Fe} 1-\mathrm{C} 3$ & $68.91(8)$ \\
\hline $\mathrm{C} 8-\mathrm{C} 9-\mathrm{H} 9$ & 125.9 & $\mathrm{C} 4-\mathrm{Fe} 1-\mathrm{C} 3$ & $40.27(8)$ \\
\hline $\mathrm{C} 10-\mathrm{C} 9-\mathrm{H} 9$ & 125.9 & $\mathrm{C} 6-\mathrm{Fe} 1-\mathrm{C} 3$ & $107.30(8)$ \\
\hline $\mathrm{Fe} 1-\mathrm{C} 9-\mathrm{H} 9$ & 125.8 & $\mathrm{C} 7-\mathrm{Fe} 1-\mathrm{C} 3$ & $125.75(8)$ \\
\hline $\mathrm{C} 9-\mathrm{C} 10-\mathrm{C} 6$ & 108.05 (19) & $\mathrm{C} 9-\mathrm{Fe} 1-\mathrm{C} 3$ & $154.59(9)$ \\
\hline $\mathrm{C} 9-\mathrm{C} 10-\mathrm{Fe} 1$ & $69.69(12)$ & $\mathrm{C} 1-\mathrm{Fe} 1-\mathrm{C} 3$ & $69.07(8)$ \\
\hline $\mathrm{C} 6-\mathrm{C} 10-\mathrm{Fe} 1$ & $69.58(12)$ & $\mathrm{C} 10-\mathrm{Fe} 1-\mathrm{C} 3$ & $119.80(9)$ \\
\hline
\end{tabular}




\begin{tabular}{|c|c|c|c|}
\hline $\mathrm{C} 9-\mathrm{C} 10-\mathrm{H} 10$ & 126.0 & $\mathrm{C} 8-\mathrm{Fe} 1-\mathrm{C} 3$ & $163.37(8)$ \\
\hline $\mathrm{C} 6-\mathrm{C} 10-\mathrm{H} 10$ & 126.0 & $\mathrm{C} 2-\mathrm{Fe} 1-\mathrm{C} 3$ & $40.09(8)$ \\
\hline $\mathrm{Fe} 1-\mathrm{C} 10-\mathrm{H} 10$ & 126.3 & & \\
\hline $\mathrm{C} 5-\mathrm{C} 1-\mathrm{C} 2-\mathrm{C} 3$ & $-0.7(2)$ & $\mathrm{C} 10-\mathrm{C} 6-\mathrm{C} 7-\mathrm{Fe} 1$ & $-59.77(15)$ \\
\hline $\mathrm{B} 1-\mathrm{C} 1-\mathrm{C} 2-\mathrm{C} 3$ & $174.20(18)$ & $\mathrm{C} 6-\mathrm{C} 7-\mathrm{C} 8-\mathrm{C} 9$ & $-0.2(2)$ \\
\hline $\mathrm{Fe} 1-\mathrm{C} 1-\mathrm{C} 2-\mathrm{C} 3$ & $58.18(14)$ & $\mathrm{Fe} 1-\mathrm{C} 7-\mathrm{C} 8-\mathrm{C} 9$ & $59.26(14)$ \\
\hline $\mathrm{C} 5-\mathrm{C} 1-\mathrm{C} 2-\mathrm{Fe} 1$ & $-58.86(13)$ & $\mathrm{C} 6-\mathrm{C} 7-\mathrm{C} 8-\mathrm{Fe} 1$ & $-59.45(14)$ \\
\hline $\mathrm{B} 1-\mathrm{C} 1-\mathrm{C} 2-\mathrm{Fe} 1$ & $116.02(18)$ & $\mathrm{C} 7-\mathrm{C} 8-\mathrm{C} 9-\mathrm{C} 10$ & $0.5(2)$ \\
\hline $\mathrm{C} 1-\mathrm{C} 2-\mathrm{C} 3-\mathrm{C} 4$ & $0.9(2)$ & $\mathrm{Fe} 1-\mathrm{C} 8-\mathrm{C} 9-\mathrm{C} 10$ & $59.65(15)$ \\
\hline $\mathrm{Fe} 1-\mathrm{C} 2-\mathrm{C} 3-\mathrm{C} 4$ & $58.44(14)$ & $\mathrm{C} 7-\mathrm{C} 8-\mathrm{C} 9-\mathrm{Fe} 1$ & $-59.18(14)$ \\
\hline $\mathrm{C} 1-\mathrm{C} 2-\mathrm{C} 3-\mathrm{Fe} 1$ & $-57.58(14)$ & $\mathrm{C} 8-\mathrm{C} 9-\mathrm{C} 10-\mathrm{C} 6$ & $-0.6(2)$ \\
\hline $\mathrm{C} 2-\mathrm{C} 3-\mathrm{C} 4-\mathrm{C} 5$ & $-0.7(2)$ & $\mathrm{Fe} 1-\mathrm{C} 9-\mathrm{C} 10-\mathrm{C} 6$ & $59.21(15)$ \\
\hline $\mathrm{Fe} 1-\mathrm{C} 3-\mathrm{C} 4-\mathrm{C} 5$ & $58.27(14)$ & $\mathrm{C} 8-\mathrm{C} 9-\mathrm{C} 10-\mathrm{Fe} 1$ & $-59.78(15)$ \\
\hline $\mathrm{C} 2-\mathrm{C} 3-\mathrm{C} 4-\mathrm{Fe} 1$ & $-58.96(14)$ & $\mathrm{C} 7-\mathrm{C} 6-\mathrm{C} 10-\mathrm{C} 9$ & $0.5(2)$ \\
\hline $\mathrm{C} 3-\mathrm{C} 4-\mathrm{C} 5-\mathrm{C} 1$ & $0.3(2)$ & $\mathrm{Fe} 1-\mathrm{C} 6-\mathrm{C} 10-\mathrm{C} 9$ & $-59.28(15)$ \\
\hline $\mathrm{Fe} 1-\mathrm{C} 4-\mathrm{C} 5-\mathrm{C} 1$ & $60.03(14)$ & $\mathrm{C} 7-\mathrm{C} 6-\mathrm{C} 10-\mathrm{Fe} 1$ & $59.73(14)$ \\
\hline $\mathrm{C} 3-\mathrm{C} 4-\mathrm{C} 5-\mathrm{C} 13$ & $-171.44(18)$ & $\mathrm{C} 4-\mathrm{C} 5-\mathrm{C} 13-\mathrm{O} 1$ & $172.7(2)$ \\
\hline $\mathrm{Fe} 1-\mathrm{C} 4-\mathrm{C} 5-\mathrm{C} 13$ & $-111.68(19)$ & $\mathrm{C} 1-\mathrm{C} 5-\mathrm{C} 13-\mathrm{O} 1$ & $2.7(3)$ \\
\hline $\mathrm{C} 3-\mathrm{C} 4-\mathrm{C} 5-\mathrm{Fe} 1$ & $-59.77(14)$ & $\mathrm{Fe} 1-\mathrm{C} 5-\mathrm{C} 13-\mathrm{O} 1$ & $88.4(2)$ \\
\hline $\mathrm{C} 2-\mathrm{C} 1-\mathrm{C} 5-\mathrm{C} 4$ & $0.3(2)$ & $\mathrm{C} 4-\mathrm{C} 5-\mathrm{C} 13-\mathrm{C} 14$ & $-7.8(3)$ \\
\hline $\mathrm{B} 1-\mathrm{C} 1-\mathrm{C} 5-\mathrm{C} 4$ & $-173.9(2)$ & $\mathrm{C} 1-\mathrm{C} 5-\mathrm{C} 13-\mathrm{C} 14$ & $-177.86(19)$ \\
\hline $\mathrm{Fe} 1-\mathrm{C} 1-\mathrm{C} 5-\mathrm{C} 4$ & $-60.11(14)$ & $\mathrm{Fe} 1-\mathrm{C} 5-\mathrm{C} 13-\mathrm{C} 14$ & $-92.1(2)$ \\
\hline $\mathrm{C} 2-\mathrm{C} 1-\mathrm{C} 5-\mathrm{C} 13$ & $171.61(19)$ & $\mathrm{C} 2-\mathrm{C} 1-\mathrm{B} 1-\mathrm{O} 3$ & $11.5(3)$ \\
\hline $\mathrm{B} 1-\mathrm{C} 1-\mathrm{C} 5-\mathrm{C} 13$ & $-2.5(4)$ & $\mathrm{C} 5-\mathrm{C} 1-\mathrm{B} 1-\mathrm{O} 3$ & $-175.2(2)$ \\
\hline $\mathrm{Fe} 1-\mathrm{C} 1-\mathrm{C} 5-\mathrm{C} 13$ & $111.3(2)$ & $\mathrm{Fe} 1-\mathrm{C} 1-\mathrm{B} 1-\mathrm{O} 3$ & $96.7(2)$ \\
\hline $\mathrm{C} 2-\mathrm{C} 1-\mathrm{C} 5-\mathrm{Fe} 1$ & $60.36(13)$ & $\mathrm{C} 2-\mathrm{C} 1-\mathrm{B} 1-\mathrm{O} 2$ & $-169.19(19)$ \\
\hline $\mathrm{B} 1-\mathrm{C} 1-\mathrm{C} 5-\mathrm{Fe} 1$ & $-113.8(2)$ & $\mathrm{C} 5-\mathrm{C} 1-\mathrm{B} 1-\mathrm{O} 2$ & $4.1(4)$ \\
\hline $\mathrm{C} 10-\mathrm{C} 6-\mathrm{C} 7-\mathrm{C} 8$ & $-0.2(2)$ & $\mathrm{Fe} 1-\mathrm{C} 1-\mathrm{B} 1-\mathrm{O} 2$ & $-84.0(2)$ \\
\hline $\mathrm{Fe} 1-\mathrm{C} 6-\mathrm{C} 7-\mathrm{C} 8$ & $59.61(14)$ & & \\
\hline
\end{tabular}

Hydrogen-bond geometry $\left(A,{ }^{\circ}\right)$

\begin{tabular}{lllll}
\hline$D-\mathrm{H} \cdots A$ & $D-\mathrm{H}$ & $\mathrm{H} \cdots A$ & $D \cdots A$ & $D-\mathrm{H} \cdots A$ \\
\hline $\mathrm{O} 2-\mathrm{H} 2 O \cdots \mathrm{O} 1$ & 0.82 & 1.85 & $2.650(2)$ & 166 \\
$\mathrm{O} 3-\mathrm{H} 3 O \cdots \mathrm{O} 2^{\mathrm{i}}$ & 0.82 & 1.94 & $2.744(2)$ & 168 \\
$\mathrm{C} 9-\mathrm{H} 9 \cdots 3^{i i}$ & 0.93 & 2.45 & $3.308(3)$ & 154 \\
$\mathrm{C} 10-\mathrm{H} 10 \cdots \mathrm{O} 1^{\mathrm{iii}}$ & 0.93 & 2.53 & $3.404(3)$ & 156
\end{tabular}

Symmetry codes: (i) $-x+1,-y+1,-z+1$; (ii) $x-1, y, z$; (iii) $x-1 / 2,-y+3 / 2, z-1 / 2$. 